\title{
Numerical Simulation of Residual Stresses Induced by Weld Repair in a Stainless Steel Pipe Considering the Influence of an Initial Fabrication Weld
}

\author{
Gervasio Salerno $^{1, a^{*}}$, Chris Bennett ${ }^{1, b}$, Wei Sun ${ }^{1, \mathrm{c}}$ and Adib A. Becker ${ }^{1, \mathrm{~d}}$ \\ ${ }^{1}$ Gas Turbine and Transmissions Research Group, Faculty of Engineering, The University of \\ Nottingham, University Park, Nottingham, NG7 2RD, UK \\ aepxgs3@nottingham.ac.uk, bchris.bennett@nottingham.ac.uk, cw.sun@nottingham.ac.uk, \\ da.a.becker@nottingham.ac.uk
}

Keywords: Finite Element Modelling, Weld Repair, Residual Stress

\begin{abstract}
This work presents the application of a finite element (FE) model developed to simulate the repair process in the case of components with a pre-existing stress state. The approach is tested in the case of a repair of a laser beam weld in a stainless steel pipe with the region of repair located in the heat affected zone of the original weld. The area of the repair is removed and refilled testing different approaches in terms of the number, and direction of the repair passes. The comparison between the refilling procedures is presented with the aim of evaluating the effects on the final residual stress distribution.
\end{abstract}

\section{Introduction}

Weld repair is commonly adopted to restore the integrity of a weld when defects are detected. According to the size and position of the defect, different repair geometries may be used, including shallow or deep and short or long excavations, resulting in different residual stress distributions. In the literature, only a few works are available where the attention was focused on the numerical simulation of weld repair. On the one hand the benefits of a 2D simulation in computational welding mechanics have been highlighted by Dong et al. [1], Brown et al. [2], Kim et al. [3] and Limpus et al. [4]: easier modelling procedure and smaller models which require lower computing power. On the other hand, Feng et al. [5] as well as Bonnaud and Gunnars [6] pointed out that only 3D simulations can capture effects due to a repair, for instance the details at start/stop locations. Brust and Rudland [7] discussed the merits and limitation of 2D versus 3D solutions including several examples of their simulations: a partial circumferential pipe repair, an industrial weld fabrication and ship structure weld analyses.

In this work, the repair of a laser beam weld in a stainless steel pipe is simulated by means of a numerical FE approach developed by Salerno et al [8] adopting 3D simulations. The area of the repair is removed and refilled testing different strategies in terms of the number and direction of the repair passes. The comparison between the refilling procedures is presented highlighting the effects on the final residual stress distribution.

\section{Case study}

A laser beam welding (LBW) was used to join two pipes made of SUS316 stainless steel to create a whole unique pipe with length $800 \mathrm{~mm}$. The work from Deng and Kiyoshima [9] was used as a reference for the simulation of the fabrication weld. The inside diameter of the pipe was $200 \mathrm{~mm}$, and the wall thickness $10 \mathrm{~mm}$. As a small defect was assumed to appear at the boundary of the HAZ and the fusion zone, the repair procedure was carried out by removing a slot $5 \mathrm{~mm}$ deep through the thickness, $5 \mathrm{~mm}$ wide and covering an arc of $40^{\circ}$, located as shown in Fig1. 

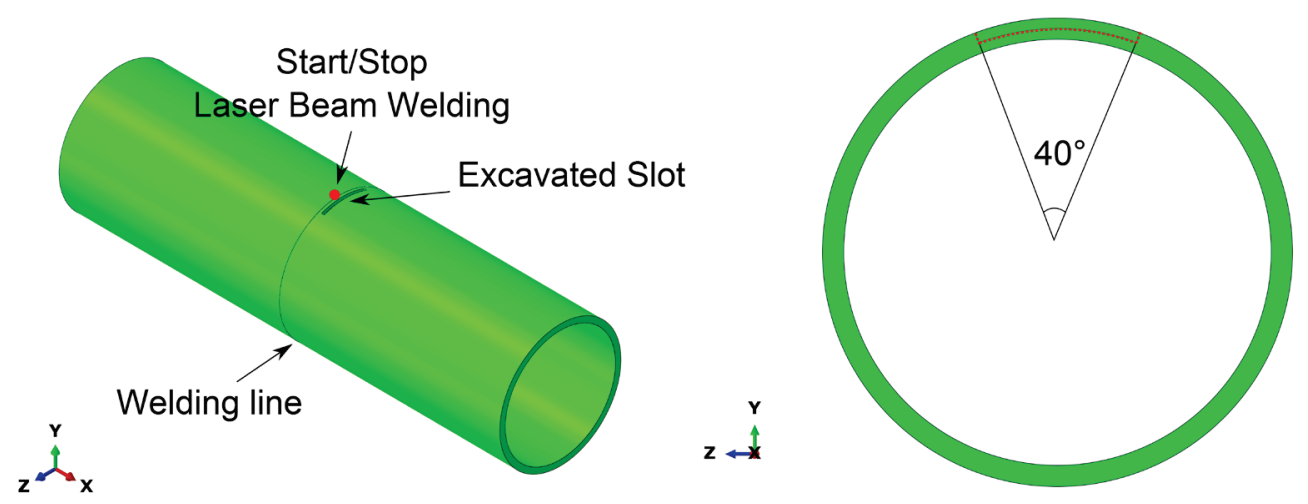

Fig. 1 Fabrication weld and excavated slot

To obtain full penetration through the wall thickness, the power of the LBW and the speed were set as $12 \mathrm{~kW}$ and $1000 \mathrm{~mm} / \mathrm{min}$, respectively as by Deng and Kiyoshima [9]. It was assumed that the slot was refilled using TIG welding. The weld power for the TIG process was set with a trial/error approach to ensure the elements simulating the filler being deposited reached the melting temperature. Different refilling approaches were investigated as described in Table 1. The terms Same Direction (SD) and Opposite Direction (OD) refer to the direction of the original LBW. In the case of two passes, these were assumed to be on top of each other, with the first one filling half the depth of the slot.

Table 1 Refilling procedure investigated

\begin{tabular}{|l|l|}
\hline Case A & One pass - Same Direction (SD) \\
\hline Case B & One pass - Opposite Direction (OD) \\
\hline Case C & Two passes OD + OD \\
\hline Case D & Two passes OD + SD \\
\hline Case E & Two passes SD + SD \\
\hline Case F & Two passes SD + OD \\
\hline
\end{tabular}

\section{FE model}

The computational procedure developed by Salerno et al. [8] was adopted in the present study using the commercial FE software Abaqus. A view of half mesh and cross section is shown in Fig. 2.
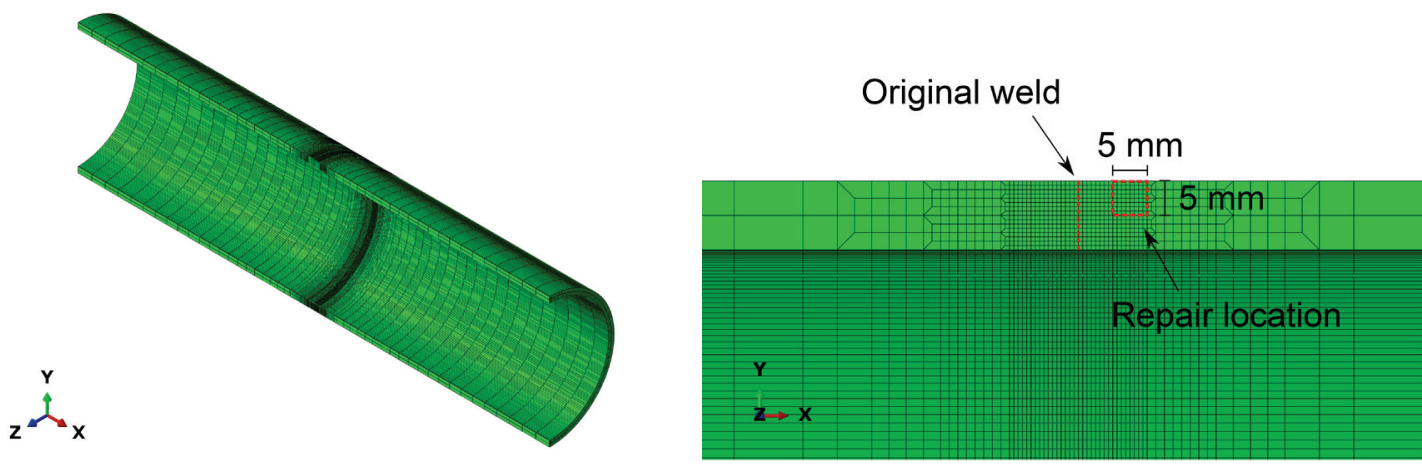

Fig. 2 Views of the FE mesh showing the transition from the weld region to the far field.

This highlights the mesh size transition adopted to decrease the number of elements, moving from the weld region to the far field. 282936 linear hexahedral elements were used for both the thermal and mechanical analyses to reduce the computational cost, yet still obtain a representative solution. The heat source adopted to simulate the initial weld was the truncated cone power density 
distribution which is commonly chosen to simulate beam welding processes while the double ellipsoid was selected for the simulation of the TIG weld repair [10]. The geometric parameters of the heat sources were chosen to produce a plausible weld pool in both cases as shown in Figs. 3 and 4.

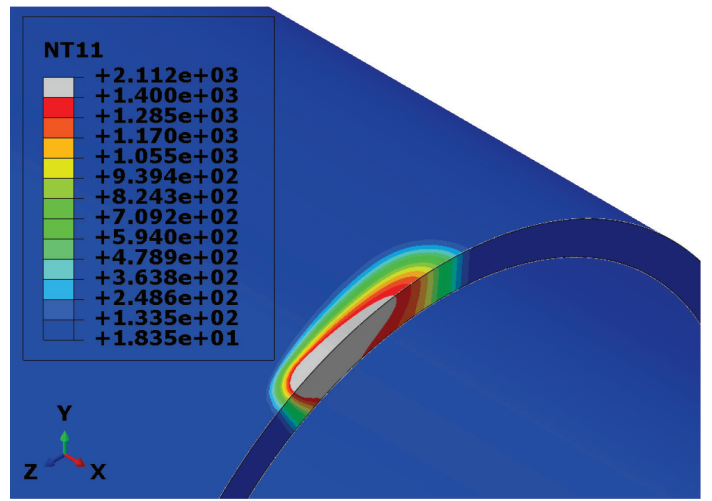

Fig. 3 Weld pool for the LBW

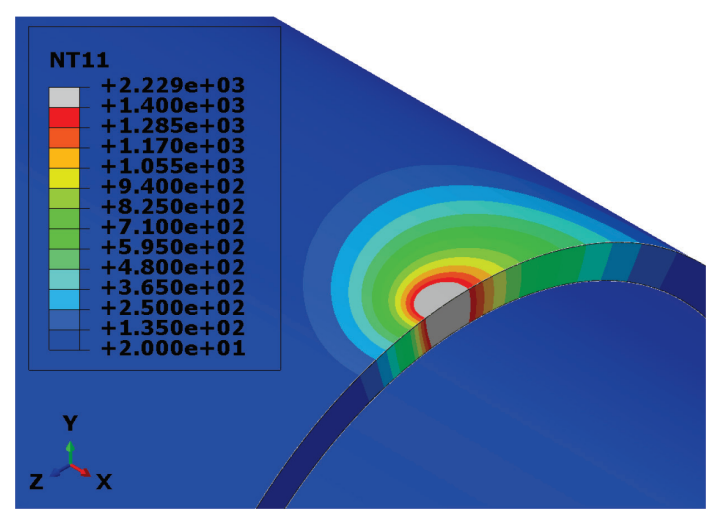

Fig. 4 Weld pool for the TIG

In the mechanical analyses, the elastic behavior was modelled using the isotropic Hooke's law while the von Mises yield criterion was adopted to determine the onset of plastic behavior. Strain hardening was taken into account using a linear isotropic hardening law. The annealing temperature option was used to simulate the loss of the hardening memory of the material. This was assumed to be $800^{\circ} \mathrm{C}$ for SUS316 stainless steel [11]. When the temperature of a material point is higher than the annealing temperature, the equivalent plastic strain is reset to zero. If the temperature of a material point falls below the annealing temperature at a subsequent point in time, the material point can work harden again. Microstructural changes were not considered in the analyses. The boundary conditions were assigned in three nodes located on the one end of the pipe to avoid rigid body motions, constraining node 1 along $\mathrm{X}, \mathrm{Y}, \mathrm{Z}$, node 2 along $\mathrm{X}, \mathrm{Y}$ and node 3 along $\mathrm{X}$.

\section{Results and discussion}

Numerical predictions for the LBW were verified by means of the numerical results from Deng and Kiyoshima [8] both in terms of thermal and mechanical analyses. In Fig.5 hoop and axial residual stresses due to the LBW and the repair procedures investigated are presented on the paths shown. The effects of the repair on the initial residual stress are evident, causing a redistribution in both the axial and hoop stress. The repair approaches investigated induce visible differences on the axial stress (Fig.5a). The highest tensile stresses in the axial distribution are regulated by the direction of the repair pass both for one and two passes. These appear approximately at the boundary of the slot being refilled $\left(70^{\circ}\right.$ and $\left.110^{\circ}\right)$ due to the restraint imposed by the surrounding material. For the same reason, lower local maxima of axial residual stress are visible at the starting location when the material is deposited in two passes. These are not present when the deposition is simulated in a single pass. In the case of two passes, the direction of the second pass governs the final distribution of the axial stress which appears to be comparable to the one obtained with a single pass in the same direction of the second one. The hoop stress is not as affected by the repair strategy adopted as the axial stress. Trends on the two axial paths considered are comparable for all the cases analyzed both for the axial and hoop (Figs.5a and 5b). Along the axial path on the O.D. the hoop stress becomes compressive because of the repair pass while it stays tensile for the path on the I.D. As highlighted in Table 2, the maximum and minimum axial and hoop stresses are not significantly influenced by the repair approach adopted. The highest axial stresses are in the case of two passes when the second pass is in the same direction of the LBW. The highest hoop stress is not due to the repair. 

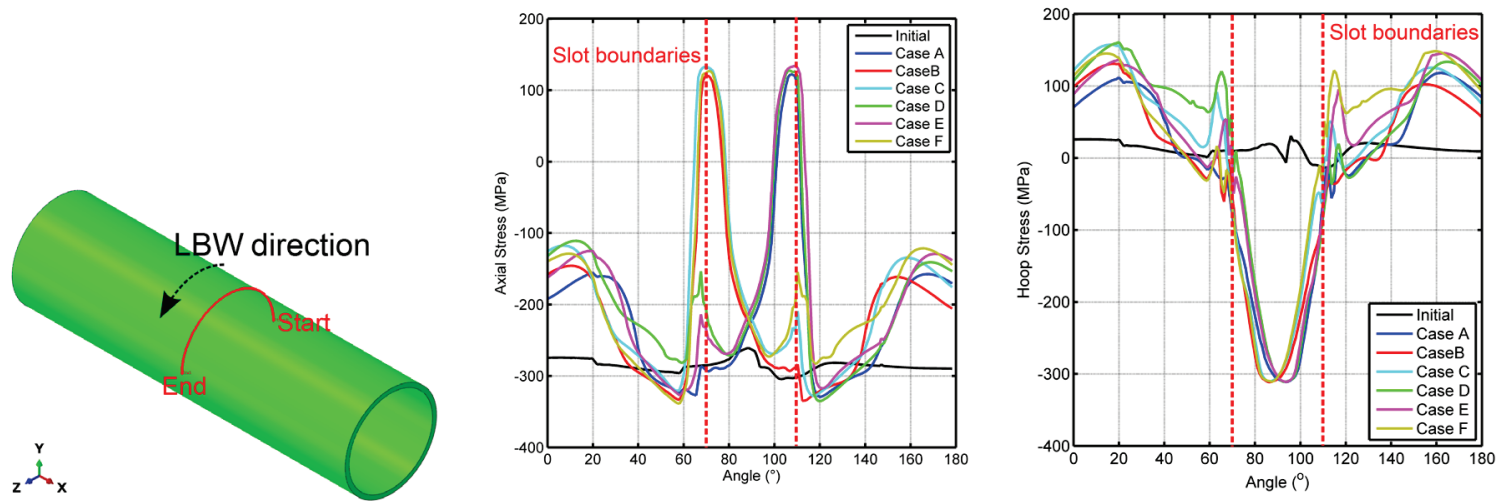

(a-Semi-circumferential path located at the centreline of the repair weld)
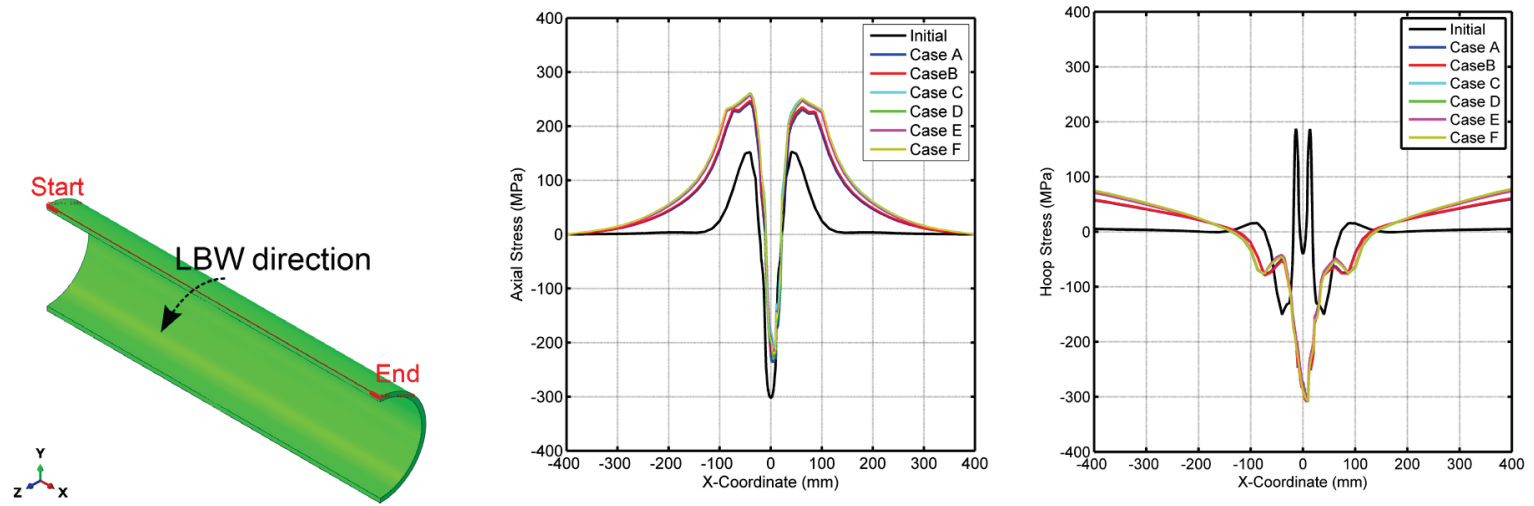

(b-Axial path located at the start of the initial weld)
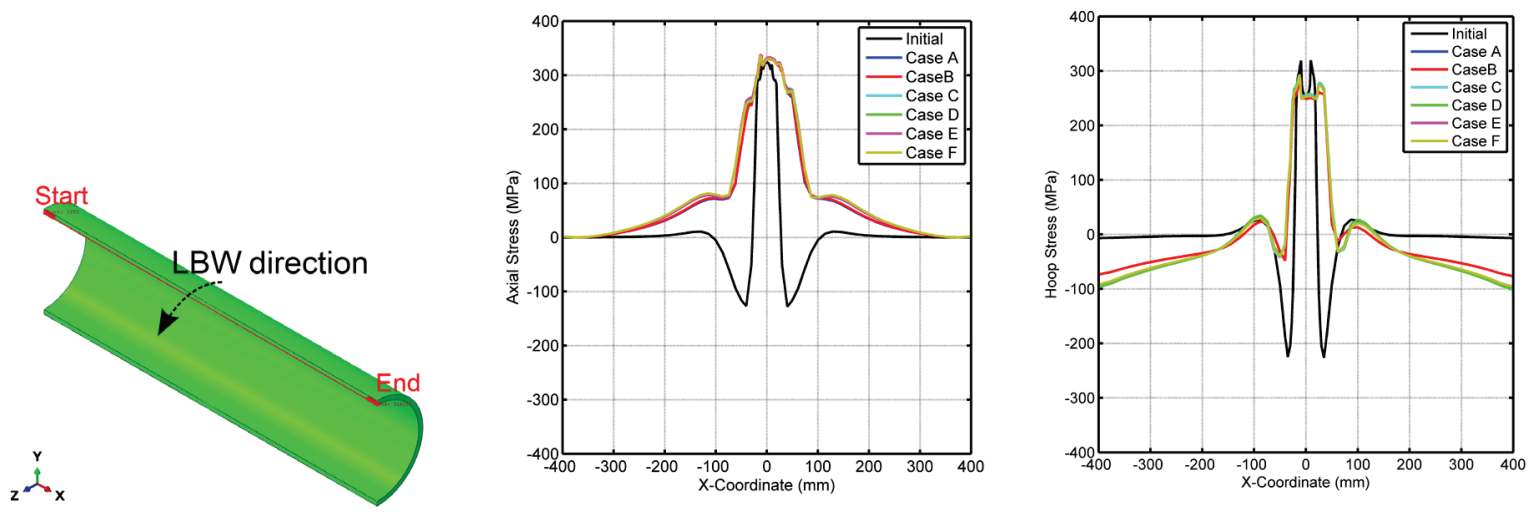

(c- Axial path located at the start of the initial weld)

Fig. 5 Axial and Hoop stress on highlighted paths. O.D. (a-b) and I.D. (c)

Table 2 Maximum and minimum axial and hoop stresses in MPa for the cases studied.

\begin{tabular}{|l|c|c|c|c|}
\hline & Axial - Max & Axial - Min & Hoop - Max & Hoop - Min \\
\hline LBW & 350 & -314 & 354 & -257 \\
\hline Case A & 410 & -353 & 354 & -320 \\
\hline Case B & 409 & -353 & 354 & -320 \\
\hline Case C & 435 & -361 & 354 & -321 \\
\hline Case D & 425 & -360 & 354 & -320 \\
\hline Case E & 439 & -361 & 354 & -321 \\
\hline Case F & 422 & -361 & 354 & -321 \\
\hline
\end{tabular}



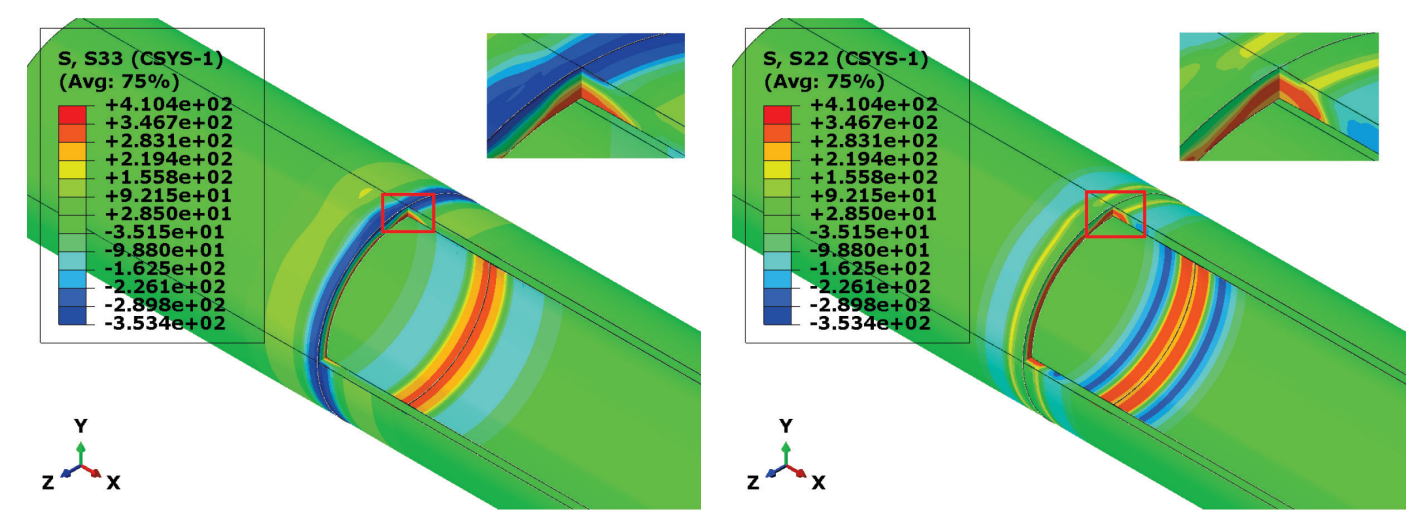

(a)
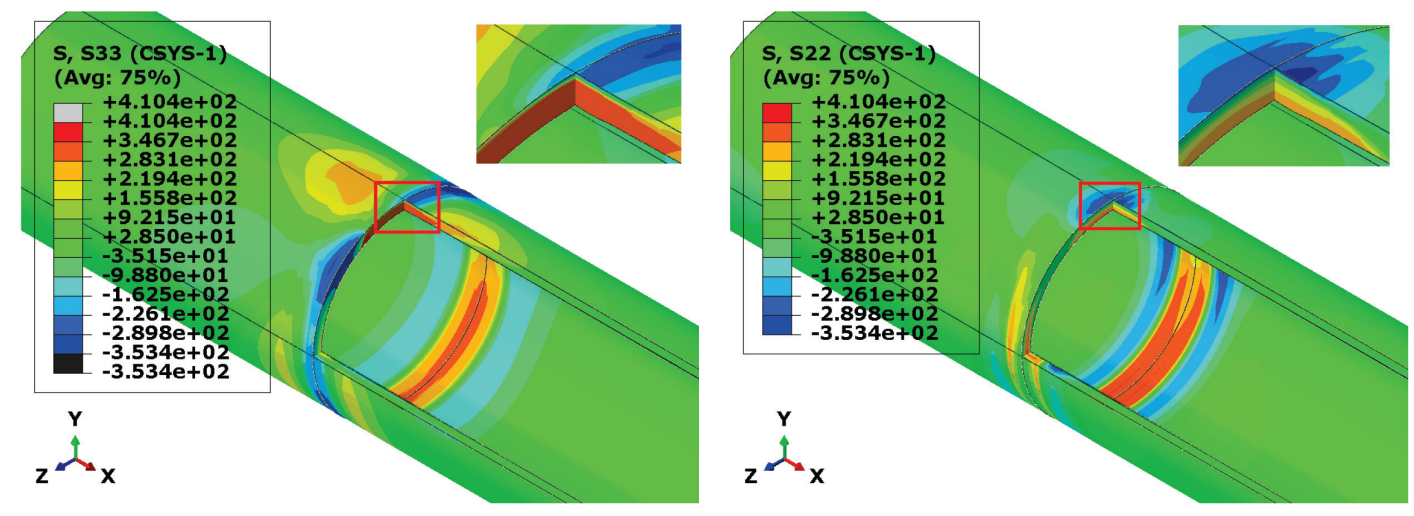

(b)

Fig. 6 Axial (left) and Hoop (right) residual stress in the pipe (Mpa): Initial residual stress (a) Case A (b)

Fig. 6 shows the distribution of the axial and hoop stress after the LBW and the case A. A quarter of the pipe was hidden for convenience to evaluate the distribution of the stress across the thickness. Tensile regions appear in the axial stress distribution because of the repair with highly compressive areas located at the start/stop area while a compressive stress is visible in the hoop distribution in the repair region. However the field results do not highlight relevant differences for the repair strategies analyzed.

\section{Conclusions and future work}

- The initial residual stress field redistributes because of the repair procedure whatever repair strategy is adopted.

- The number of passes and the deposition sequence simulated do not appear to have a significant effect on the magnitude of residual stress, both for the axial and the hoop stress. The numerical model suggests there is no relevant benefit of using a repair strategy or another in terms of lowering the maximum tensile stress.

- The distribution of the hoop stress is less affected by the number of passes and their direction compared to the axial stress.

- In the case of two passes the second pass governs the distribution of the final axial stress. This appears very similar to the one obtained with a single pass in the same direction as the second one. Therefore, if the aim of the analysis is to obtain a quicker, yet still representative solution, it is sufficient to simulate the material deposition in a single step with the deposition direction of the final pass. 
Future work will include the investigation of different slot geometries and refilling strategies, welding power for the repair procedure and the microstructural evolution driven by the weld passes on the material properties.

\section{Acknowledgments}

The authors wish to thank Rolls-Royce plc for their financial support of the research, which was carried out at the University Technology Centre in Gas Turbine Transmission Systems at The University of Nottingham. The views expressed in this paper are those of the authors and not necessarily those of Rolls-Royce plc. The authors are also grateful for access to the University of Nottingham High Performance Computing (HPC) Facility.

\section{References}

[1] Dong, P. and Hong, J.K. and Bouchard, P.J., Analysis of residual stresses at weld repairs, International journal of pressure vessels and piping 82 (2005) 258-269. http://dx.doi.org/10.1016/j.ijpvp.2004.08.004

[2] Brown, T.B. and Dauda, T.A. and Truman, C.E. and Smith, D.J. and Memhard, D. and Pfeier, W., Predictions and measurements of residual stress in repair welds in plates, International journal of pressure vessels and piping 83 (2006) 809-818. http://dx.doi.org/10.1016/j.ijpvp.2006.08.012

[3] Kim, K.S., Lee, H.J., Lee, B.S. Jung, I.C. and Park, K.S. Residual stress analysis of an Overlay weld and a repair weld on the dissimilar Butt weld. Nuclear Engineering and Design 239.12 (2009): 2771-2777. http://dx.doi.org/10.1016/j.nucengdes.2009.08.022

[4] Limpus, C. R., et al. Effect of size of butt weld repairs on weld overlay residual stresses. ASME 2007 Pressure Vessels and Piping Conference. American Society of Mechanical Engineers, 2007. http://dx.doi.org/10.1115/pvp2007-26636

[5] Feng, Z. and Wang, X.L. and Spooner, S. and Goodwin, G.M. and Maziasz, P.J. and Hubbard, C.R. and Zacharia, T., A finite element model for residual stress in repair welds, Technical Report, Oak Ridge National Lab., TN (United States), 1996. http://dx.doi.org/10.2172/244602

[6] Bonnaud, E., and J. Gunnars. Three Dimensional Weld Residual Stresses Simulations of Start/Stop and Weld Repair Effects. Procedia Engineering 130 (2015): 531-543. http://dx.doi.org/10.1016/j.proeng.2015.12.260

[7] Brust, F. W., and Rudland, D. L. Three dimensional aspects of computational weld modelling. ASME 2008 Pressure Vessels and Piping Conference. American Society of Mechanical Engineers, 2008. http://dx.doi.org/10.1115/pvp2008-61558

[8] Salerno, G., Bennett, C.J., Sun, W. and Becker, A.A., FE modelling strategies for weld repair in pre-stressed thin components: submitted to Journal of Strain Analysis for Engineering Design (2016). http://dx.doi.org/10.1177/0309324716666436

[9] Deng, D., and Kiyoshima, S. Numerical simulation of residual stresses induced by laser beam welding in a SUS316 stainless steel pipe with considering initial residual stress influences. Nuclear Engineering and Design 240.4 (2010): 688-696. http://dx.doi.org/10.1016/j.nucengdes.2009.11.049

[10] Lacki, P., and Konrad, A. Numerical simulation of the electron beam welding process. Computers \& Structures 89.11 (2011): 977-985.

[11] Ogawa, K. and Deng, D. Influences of material properties and FEM elements on welding residual stress analysis. Symposium of Welded Structure, Osaka, Japan. Vol. 4. 2006.

http://dx.doi.org/10.1016/j.compstruc.2011.01.016 